\title{
Final amendment: A plausible explanation for in silico reporting of erroneous MET gene expression in tumor-educated platelets (TEP) intended for "liquid biopsy" of non-small cell lung carcinoma still refutes the TEP-study \\ Sandeep Chakraborty, R - 44/ 1, Celia Engineers, T. T. C Industrial Area, Rabale, Navi Mumbai, 400701, India.
}

\begin{abstract}
Final amendment note: This paper had proposed a plausible way for detecting large quantities of MET, which the authors have clarified was not done :the possible explanation proposed for this erroneous MET gene expression does bypass the filtering step we perform in the data processing pipeline, i.e. selection of intron-spanning reads, as can be read in the main text" comments in http://www.biorxiv.org/content/early/2017/07/02/146134, where a continuing critique of the TEP study continues. Please consider this pre-print closed.

Original abstract:

The reported over-expression of MET genes in non-small cell lung carcinoma (NSCLC) from an analysis of the RNA-seq data from tumor-educated platelets (TEP), intended to supplement existing 'liquid biopsy' techniques [1], has been refuted recently (http://biorxiv.org/content/early/2017/06/05/146134, not peer-reviewed). The MET proto-oncogene (Accid:NG_008996.1, RefSeqGene LRG_662 on chromosome 7, METwithintrons) encodes 21 exons resulting in a 6710 bps MET gene (Accid: NM_001127500.2, METonlyexons). METwithintrons has multiple matches in the RNA-seq derived reads of lung cancer samples (for example: SRR1982756.11853382). Unfortunately, these are non-specific sequences in the intronic regions, matching to multiple genes on different chromosomes with $100 \%$ identity (KIF6 on chr6, COL6A6 on chr3, MYO16 on chr13, etc. for SRR1982756.11853382). In contrast, METonlyexons has few matches in the reads, if at all [2]. However, even RNA-seq from healthy donors have similar matches for METwithintrons - so the computation behind the over-expression statistic remains obscure, even if METwithintrons was used as the search gene. In summary, this work re-iterates the lack of reproducibility in the bioinformatic analysis that establishes TEP as a possible source for "liquid biopsy".
\end{abstract}




\section{Introduction}

Tumor tissue biopsy, the gold standard for cancer diagnostics, pose challenges that include access to the tumor, quantity and quality of tumoral material, lack of patient compliance, repeatability, and bias of sampling a specfic area of a single tumor [3]. This has resulted in a new medical and scientific paradigm defined by minimal invasiveness, high-efficiency, low-cost diagnostics [4], and, whenever possible, personalized treatment based on genetic and epigenetic composition [5]. The presence of fragmented DNA in the cell-free component of whole blood (cfDNA) [6], first reported in 1948 by Mandel and Metais, has been extensively researched for decades, with extremely promising results in certain niches [7]. Additionally, cfDNA derived from tumors (ctDNA) [8] have tremendous significance as a cancer diagnostic tool [9], and for monitoring responses to treatment [10]. However, detection of ctDNA, and differentiation with cfDNA, remains a challenge due the low amounts of ctDNA compared to cfDNA [11].

Recently, tumor-educated blood platelets (TEP) were proposed as an alternative source of tumor-related biological information $[1,12]$. The hypothesis driving the potential diagnostic role of TEPs is based on the interaction between blood platelets and tumor cells, subsequently altering the RNA profile of platelets [13,14]. The study showed using RNA-seq data that tumor-educated platelets (TEP) can distinguish 228 patients with localized and metastasized tumors from 55 healthy individuals with $96 \%$ accuracy [1]. As validation, this study reported significant over-expression of MET genes in non-small cell lung carcinoma (NSCLC), and HER2/ERBB2 [15] genes in breast cancer, which are well-established biomarkers.

Previously, the TEP-study was refuted by an analysis of a subset of the samples (yet to be peerreviewed) [2]. Here, an analysis based on the complete MET gene (both introns and exons, Accid:NG_008996.1) demonstrates that intronic non-specific sequences might mislead bioinformatic analysis. Moreover, considering that RNA-seq from healthy donors have similar matches with the complete MET gene, the computation behind the over-expression statistic remains obscure [1].

\section{Results}

The MET proto-oncogene (Accid:NG_008996.1, RefSeqGene LRG_662 on chromosome 7, METWITHINTRONS) encodes 21 exons leading to a 6710 bps MET gene (Accid: NM_001127500.2, METONLYEXONS). METWITHINTRONS has multiple matches in the RNA-seq derived reads (for example: SRR1982756.11853382) (Fig. 1). Unfortunately, these are non-specific sequences in the intronic regions: SRR1982756.M.11853382 (CTTCACGTAGTTCTCGAGCCTTGGTTTTCAGCTCCATCAGCTCCTTTAAGCACTTCTCTGTA TTGGTTATTCTAGTTATACATTCTTCTAAATTTTTTTCA) matches to mutliple genes on different chromosomes (KIF6 on chr6, COL6A6 on chr3, MYO16 on chr13, etc). In contrast, METONLYEXONS has few matches in the reads, if at all [2]. Thus, it is erroneous to assign the intronic sequences being expressed to the MET gene. However, even RNA-seq from healthy donors have similar matches for METWITHINTRONS (Fig 3) - so the computation behind the over-expression statistic remains obscure, even if METwithintrons was used as the search gene.

\section{Conclusion}

Here, the absence of MET over-expression as reported in the TEP-study [1] is investigated in further detail by using the full MET gene including introns. It turns out that several intronic sequences have matches in the RNA-seq samples. However, these intronic sequences are non-specific (i.e. matching to several other genes with $100 \%$ identity). Further, there is to be large number of matches in healthy donor samples as well. This work re-iterates the lack of reproducibility in the bioinformatic analysis that establishes TEP as a possible source for "liquid biopsy". 


\section{Materials and methods}

The BLAST interface suffices to demonstrate the presence of non-specific sequences from the intronic regions of the MET gene to the RNA-seq samples, and the non-specific nature of these sequences based on $100 \%$ identity to a plethora of genes in different chromosomes. These have been verified by a kmer-based version (KEATS [16]) of YeATS [17-21], as well.

\section{Competing interests}

No competing interests were disclosed.

\section{References}

1. Best MG, Sol N, Kooi I, Tannous J, Westerman BA, et al. (2015) Rna-seq of tumor-educated platelets enables blood-based pan-cancer, multiclass, and molecular pathway cancer diagnostics. Cancer cell 28: $666-676$.

2. Chakraborty S (2017) No evidence of met and her2 over-expression in non-small cell lung carcinoma and breast cancer, respectively, raises serious doubts on using rna-seq profiles of tumor-educated platelets as a liquid biopsysource. bioRxiv : 146134 .

3. Vendrell JA, Mau-Them FT, Béganton B, Godreuil S, Coopman P, et al. (2017) Circulating cell free tumor dna detection as a routine tool forlung cancer patient management. International Journal of Molecular Sciences 18: 264.

4. Han X, Wang J, Sun Y (2017) Circulating tumor dna as biomarkers for cancer detection. Genomics, proteomics \& bioinformatics .

5. Sorber L, Zwaenepoel K, Deschoolmeester V, Van Schil P, Van Meerbeeck J, et al. (2016) Circulating cell-free nucleic acids and platelets as a liquid biopsy in the provision of personalized therapy for lung cancer patients. Lung Cancer .

6. Jiang P, Lo YD (2016) The long and short of circulating cell-free dna and the ins and outs of molecular diagnostics. Trends in Genetics 32: 360-371.

7. Lo YD, Corbetta N, Chamberlain PF, Rai V, Sargent IL, et al. (1997) Presence of fetal dna in maternal plasma and serum. The Lancet 350: 485-487.

8. Chen XQ, Stroun M, Magnenat JL, Nicod LP, Kurt AM, et al. (1996) Microsatellite alterations in plasma dna of small cell lung cancer patients. Nature medicine 2: 1033-1035.

9. Yi X, Ma J, Guan Y, Chen R, Yang L, et al. (2017) The feasibility of using mutation detection in ctdna to assess tumor dynamics. International Journal of Cancer 140: 2642-2647.

10. Imamura F, Uchida J, Kukita Y, Kumagai T, Nishino K, et al. (2016) Monitoring of treatment responses and clonal evolution of tumor cells by circulating tumor dna of heterogeneous mutant egfr genes in lung cancer. Lung Cancer 94: 68-73.

11. Diaz LA, Bardelli A (2014) Liquid biopsies: genotyping circulating tumor dna. Journal of Clinical Oncology 32: 579-586.

12. Nilsson RJA, Balaj L, Hulleman E, Van Rijn S, Pegtel DM, et al. (2011) Blood platelets contain tumor-derived rna biomarkers. Blood 118: 3680-3683. 
13. Bardelli A, Pantel K (2017) Liquid biopsies, what we do not know (yet). Cancer cell 31: 172-179.

14. Feller SM, Lewitzky M (2016) Hunting for the ultimate liquid cancer biopsy-let the tep dance begin. Cell Communication and Signaling 14: 24.

15. Foulkes WD, Stefansson IM, Chappuis PO, Bégin LR, Goffin JR, et al. (2003) Germline brca1 mutations and a basal epithelial phenotype in breast cancer. Journal of the National Cancer Institute 95: $1482-1485$.

16. Chakraborty S (2017) Cataloguing over-expressed genes in epstein barr virus immortalized lymphoblastoid cell lines through consensus analysis of pacbio transcriptomes corroborates hypomethylation of chromosome 1. bioRxiv : 125823.

17. Chakraborty S, Britton M, Wegrzyn J, Butterfield T, Martinez-Garcia PJ, et al. (2015). YeATS-a tool suite for analyzing RNA-seq derived transcriptome identifies a highly transcribed putative extensin in heartwood/sapwood transition zone in black walnut.

18. Martínez-García PJ, Crepeau MW, Puiu D, Gonzalez-Ibeas D, Whalen J, et al. (2016) The walnut (juglans regia) genome sequence reveals diversity in genes coding for the biosynthesis of nonstructural polyphenols. The Plant Journal .

19. Chakraborty S, Britton M, Martínez-García P, Dandekar AM (2016) Deep RNA-seq profile reveals biodiversity, plant-microbe interactions and a large family of NBS-LRR resistance genes in walnut (juglans regia) tissues. AMB Express 6: 1.

20. Chakraborty S, Martínez-García PJ, Dandekar AM (2016) Yeatsam analysis of the walnut and chickpea transcriptome reveals key genes undetected by current annotation tools. F1000Research 5 .

21. Chakraborty S (2017) Mcf-7 breast cancer cell line pacbio generated transcriptome has ${ }^{\sim} 300$ novel transcribed regions, un-annotated in both refseq and gencode, and absent in the liver, heart and brain transcriptomes. bioRxiv : 100974. 
bioRxiv preprint doi: https://doi.org/10.1101/148718; this version posted July 18,2017 . The copyright holder for this preprint (which was not certified by peer review) is the author/funder, who has granted bioRxiv a license to display the preprint in perpetuity. It is made available under aCC-BY-NC-ND 4.0 International license. 
Table 1: Raw counts of reads matching to the MET gene: Although this is a subset (24 out of 60 NSCLC, and 20 out of 60 healthy), and the numbers are not normalized, its seems unlikely that any statistic will show MET over-expression in NSCLC. It does not even add up to one complete gene in most cases.

\begin{tabular}{|c|c|c|c|}
\hline Healthy & $\begin{array}{l}\text { SRR1982752 } \\
\text { SRR1982731 } \\
\text { SRR1982742 } \\
\text { SRR1982702 } \\
\text { SRR1982750 } \\
\text { SRR1982741 } \\
\text { SRR1982720 } \\
\text { SRR1982730 } \\
\text { SRR1982722 } \\
\text { SRR1982735 } \\
\text { SRR1982740 } \\
\text { SRR1982751 } \\
\text { SRR2095004 } \\
\text { SRR1982721 } \\
\text { SRR1982732 } \\
\text { SRR1982737 } \\
\text { SRR1982700 } \\
\text { SRR2095014 } \\
\text { SRR1982710 } \\
\text { SRR1982738 } \\
\text { SRR1982701 }\end{array}$ & $\begin{array}{l}\text { NM_001127500.2 } \\
\text { NM_001127500.2 } \\
\text { NM_001127500.2 } \\
\text { NM_001127500.2 } \\
\text { NM_001127500.2 } \\
\text { NM_001127500.2 } \\
\text { NM_001127500.2 } \\
\text { NM_001127500.2 } \\
\text { NM_001127500.2 } \\
\text { NM_001127500.2 } \\
\text { NM_001127500.2 } \\
\text { NM_001127500.2 } \\
\text { NM_001127500.2 } \\
\text { NM_001127500.2 } \\
\text { NM_001127500.2 } \\
\text { NM_001127500.2 } \\
\text { NM_001127500.2 } \\
\text { NM_001127500.2 } \\
\text { NM_001127500.2 } \\
\text { NM_001127500.2 } \\
\text { NM_001127500.2 }\end{array}$ & $\begin{aligned} \text { ntranscripts } & =278+2 \\
\text { ntranscripts } & =194+0 \\
\text { ntranscripts } & =56+2 \\
\text { ntranscripts } & =127+2 \\
\text { ntranscripts } & =174+2 \\
\text { ntranscripts } & =124+2 \\
\text { ntranscripts } & =7+0 \\
\text { ntranscripts } & =171+0 \\
\text { ntranscripts } & =2+0 \\
\text { ntranscripts } & =132+0 \\
\text { ntranscripts } & =179+2 \\
\text { ntranscripts } & =152+2 \\
\text { ntranscripts } & =23+0 \\
\text { ntranscripts } & =2+0 \\
\text { ntranscripts } & =180+2 \\
\text { ntranscripts } & =167+2 \\
\text { ntranscripts } & =78+2 \\
\text { ntranscripts } & =23+0 \\
\text { ntranscripts } & =28+0 \\
\text { ntranscripts } & =69+2 \\
\text { ntranscripts } & =99+3\end{aligned}$ \\
\hline NSCLC & $\begin{array}{l}\text { SRR1982781 } \\
\text { SRR1982780 } \\
\text { SRR1982791 } \\
\text { SRR2096517 } \\
\text { SRR1982772 } \\
\text { SRR1982770 } \\
\text { SRR1982790 } \\
\text { SRR2096502 } \\
\text { SRR1982756 } \\
\text { SRR1982759 } \\
\text { SRR1982795 } \\
\text { SRR1982762 } \\
\text { SRR1982761 } \\
\text { SRR2096503 } \\
\text { SRR2096501 } \\
\text { SRR1982782 } \\
\text { SRR2096516 } \\
\text { SRR1982777 } \\
\text { SRR1982793 } \\
\text { SRR1982765 } \\
\text { SRR1982771 } \\
\text { SRR1982787 } \\
\text { SRR1982792 } \\
\text { SRR1982760 }\end{array}$ & $\begin{array}{l}\text { NM_001127500.2 } \\
\text { NM_001127500.2 } \\
\text { NM_001127500.2 } \\
\text { NM_001127500.2 } \\
\text { NM_001127500.2 } \\
\text { NM_001127500.2 } \\
\text { NM_001127500.2 } \\
\text { NM_001127500.2 } \\
\text { NM_001127500.2 } \\
\text { NM_001127500.2 } \\
\text { NM_001127500.2 } \\
\text { NM_001127500.2 } \\
\text { NM_001127500.2 } \\
\text { NM_001127500.2 } \\
\text { NM_001127500.2 } \\
\text { NM_001127500.2 } \\
\text { NM_001127500.2 } \\
\text { NM_001127500.2 } \\
\text { NM_001127500.2 } \\
\text { NM_001127500.2 } \\
\text { NM_001127500.2 } \\
\text { NM_001127500.2 } \\
\text { NM_001127500.2 }\end{array}$ & $\begin{aligned} \text { ntranscripts } & =13+2 \\
\text { ntranscripts } & =24+0 \\
\text { ntranscripts } & =5+0 \\
\text { ntranscripts } & =8+4 \\
\text { ntranscripts } & =8+2 \\
\text { ntranscripts } & =44+2 \\
\text { ntranscripts } & =1+0 \\
\text { ntranscripts } & =5+0 \\
\text { ntranscripts } & =10+0 \\
\text { ntranscripts } & =9+0 \\
\text { ntranscripts } & =11+2 \\
\text { ntranscripts } & =5+0 \\
\text { ntranscripts } & =2+0 \\
\text { ntranscripts } & =33+2 \\
\text { ntranscripts } & =2+2 \\
\text { ntranscripts } & =6+2 \\
\text { ntranscripts } & =40+2 \\
\text { ntranscripts } & =6+0 \\
\text { ntranscripts } & =16+0 \\
\text { ntranscripts } & =11+2 \\
\text { ntranscripts } & =2+0 \\
\text { ntranscripts } & =5+0 \\
\text { ntranscripts } & =2+0\end{aligned}$ \\
\hline
\end{tabular}




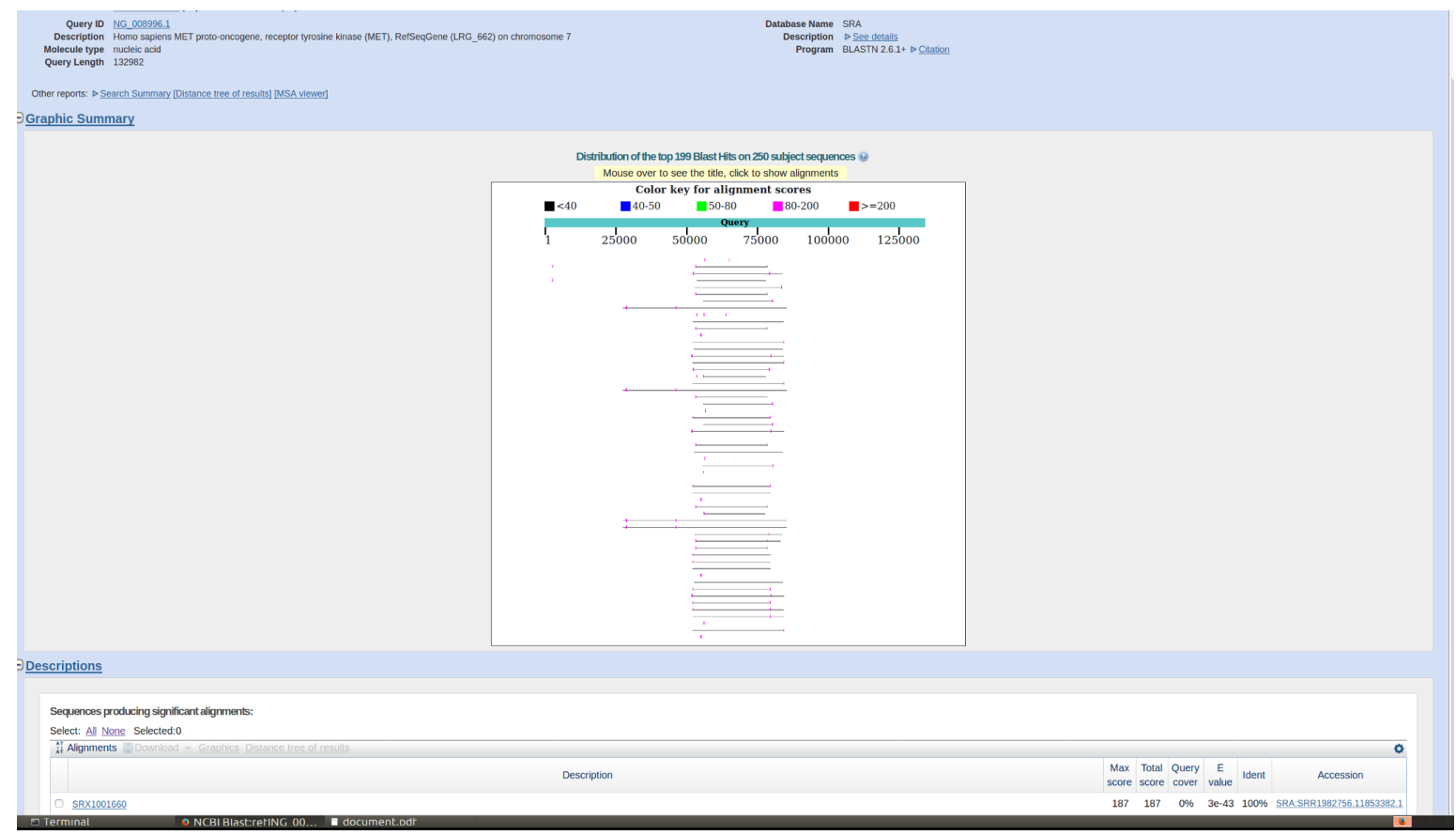

Figure 1: Graphical representation of matches to the complete MET gene (including introns) to a lung cancer sample (SRR1982756): This shows the first 100 matches, out of thousands of significant matches using the online BLAST interface. However, as demonstrated previously [2], very few of these matches are in the exonic region. Also, these sequences (an example is SRR1982756.11853382) are very non-specific and match to many genes in different chromosomes (Fig 2). 
bioRxiv preprint doi: https:/doi.org/10.1101/148718; this version posted July 182017 . The copyright holder for this preprint (which was not certified by peer review) is the author/funder, who has granted bioRxiv a license to display the preprint in perpetuity. It is made available under aCC-BY-NC-ND 4.0 International license.

\begin{tabular}{|c|c|c|c|c|c|c|c|}
\hline & Description & $\begin{array}{l}\text { Max } \\
\text { score }\end{array}$ & $\begin{array}{l}\text { Total } \\
\text { score }\end{array}$ & $\begin{array}{l}\text { Query } \\
\text { cover }\end{array}$ & $\begin{array}{c}E \\
\text { value }\end{array}$ & Ident & Accession \\
\hline$\square$ & Homo sapiens kinesin family member 6 (KIF6), RefSeqGene on chromosome 6 & 187 & 358 & $100 \%$ & $3 e-44$ & $100 \%$ & NG 054928.1 \\
\hline - & Homo sapiens collagen type VI alpha 6 chain (COL6A6), RefSeqGene on chromosome 3 & 187 & 187 & $100 \%$ & $3 e-44$ & $100 \%$ & NG 054914.1 \\
\hline 0 & Homo sapiens uveal autoantigen with coiled-coil domains and ankyrin repeats (UACA), RefSe & 187 & 187 & $100 \%$ & $3 e-44$ & $100 \%$ & NG 054898.1 \\
\hline 0 & Homo sapiens NLR family pyrin domain containing 11 (NLRP11), RefSeqGene on chromoson & 187 & 187 & $100 \%$ & $3 e-44$ & $100 \%$ & NG 054722.1 \\
\hline 0 & Homo sapiens $12 \mathrm{p} 13$ proximal LINE-mediated recombination region (LOC108178987) on chrc & $\leqslant 187$ & 187 & $100 \%$ & $3 e-44$ & $100 \%$ & NG 050935.1 \\
\hline$\circ$ & Homo sapiens 11p14.2 proximal LINE-mediated recombination region (LOC108178984) on cr & 187 & 187 & $100 \%$ & $3 e-44$ & $100 \%$ & NG 050933.1 \\
\hline$\square$ & Homo sapiens 9q21.12 distal LINE-mediated recombination region (LOC108175350) on chror & 187 & 187 & $100 \%$ & $3 e-44$ & $100 \%$ & NG 050923.1 \\
\hline$\square$ & Homo sapiens 9q21.12 proximal LINE-mediated recombination region (LOC108175349) on ch & 187 & 187 & $100 \%$ & $3 e-44$ & $100 \%$ & NG 050922.1 \\
\hline$\square$ & Homo sapiens diacylglycerol kinase beta (DGKB), RefSeqGene on chromosome 7 & 187 & 730 & $100 \%$ & $3 e-44$ & $100 \%$ & NG 029494.2 \\
\hline$\square$ & $\underline{\text { Homo sapiens myosin XVI (MYO16), RefSeqGene on chromosome } 13}$ & 187 & 187 & $100 \%$ & $3 e-44$ & $100 \%$ & NG 053147.1 \\
\hline$\square$ & Homo sapiens collagen type XXIV alpha 1 chain (COL24A1), RefSeqGene on chromosome 1 & 187 & 557 & $100 \%$ & $3 e-44$ & $100 \%$ & NG 053093.1 \\
\hline ○ & Homo sapiens regulating synaptic membrane exocytosis 2 (RIMS2), RefSeqGene on chromos & $£ 187$ & 369 & $100 \%$ & $3 e-44$ & $100 \%$ & NG 053027.1 \\
\hline$\circ$ & Homo sapiens neurexin 3 (NRXN3), RefSeqGene on chromosome 14 & 187 & 369 & $100 \%$ & $3 e-44$ & $100 \%$ & NG 052991.1 \\
\hline$\circ$ & Homo sapiens POU class 6 homeobox 2 (POU6F2), RefSeqGene on chromosome 7 & 187 & 187 & $100 \%$ & $3 e-44$ & $100 \%$ & NG 016022.2 \\
\hline - & Homo sapiens potassium voltage-gated channel interacting protein 4 (KCNIP4), RefSeqGene & 187 & 187 & $100 \%$ & $3 e-44$ & $100 \%$ & NG 052969.1 \\
\hline o & Pongo abelii genomic sequence & 187 & 722 & $100 \%$ & $3 e-44$ & $100 \%$ & $\underline{K \times 224531.1}$ \\
\hline
\end{tabular}

Figure 2: Non-specific nature of the intronic sequences of the MET gene that match to the RNA-seq reads from the platelets: The sequence SRR1982756.M.11853382 matches to several genes across different chromosomes - KIF6 on chr6, COL6A6 on chr3, MYO16 on chr13, etc.. 


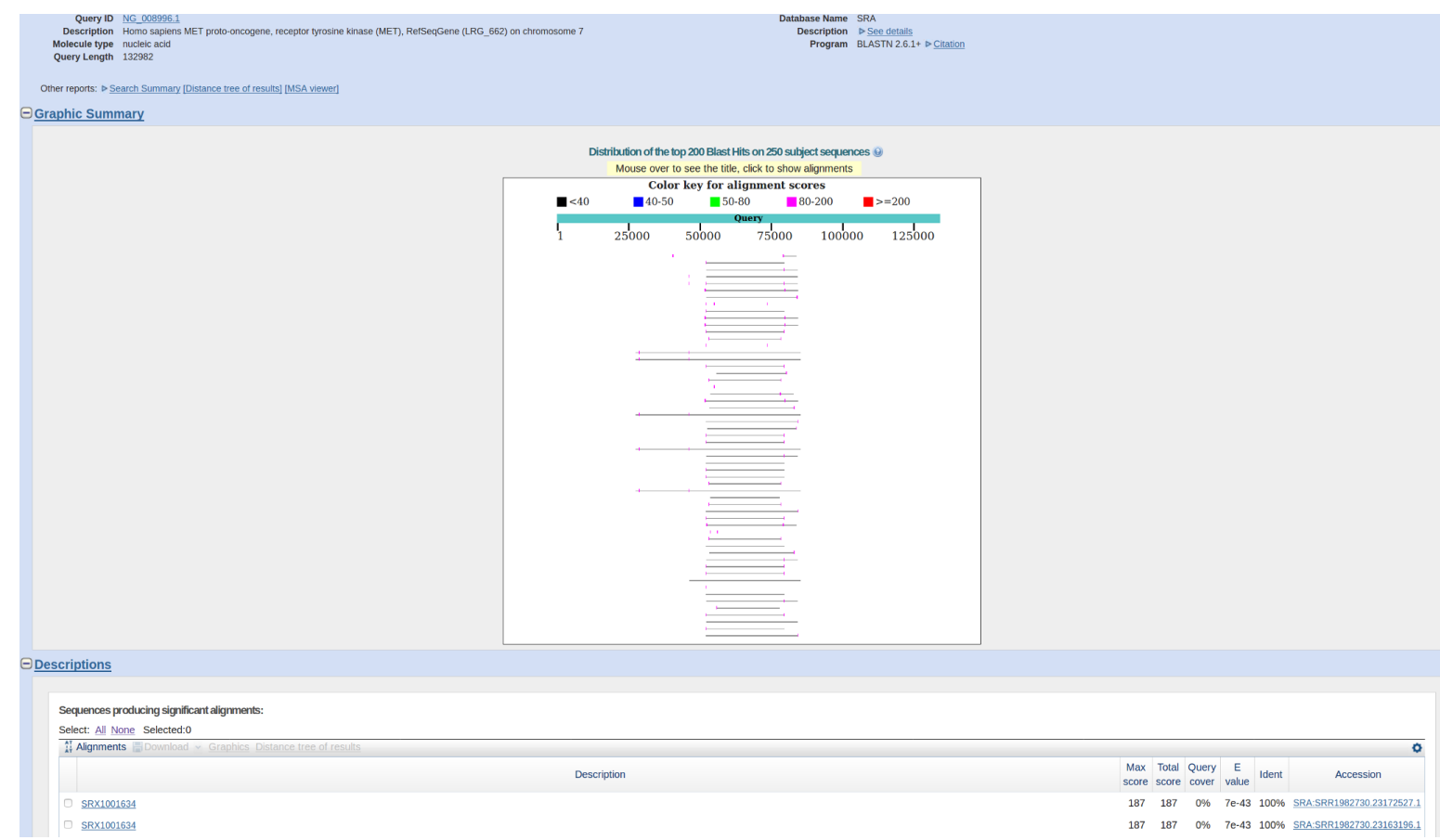

Figure 3: Graphical representation of matches to the complete MET gene (including introns) to a healthy donor sample (SRR1982730): This shows the first 100 matches, out of thousands of significant matches, as found in lung cancer samples as well (Fig. 1). Thus, any statistic showing over-expression must be validated against these raw numbers. These sequences are very non-specific and match to many genes in different chromosomes (Fig 2). 